\title{
The promastigote surface antigen gene family of the Leishmania parasite: differential evolution by positive selection and recombination
}

\author{
Alain Devault*1,2 and Anne-Laure Bañuls ${ }^{1}$
}

Address: ${ }^{1}$ Génétique et Evolution des Maladies Infectieuses, IRD/CNRS (UMR 2724), Montpellier F-34394, France and ²CRBM-CNRS (UMR5237), Montpellier F-34293, France

Email: Alain Devault* - devault@crbm.cnrs.fr; Anne-Laure Bañuls - Anna-Laure.Banuls@mpl.ird.fr

* Corresponding author

Published: 24 October 2008

BMC Evolutionary Biology 2008, 8:292 doi:10.1 186/1471-2/48-8-292
Received: 21 April 2008

Accepted: 24 October 2008

This article is available from: http://www.biomedcentral.com/I47|-2|48/8/292

(c) 2008 Devault and Bañuls; licensee BioMed Central Ltd.

This is an Open Access article distributed under the terms of the Creative Commons Attribution License (http://creativecommons.org/licenses/by/2.0), which permits unrestricted use, distribution, and reproduction in any medium, provided the original work is properly cited.

\begin{abstract}
Background: PSA (promastigote surface antigen) is one of the major classes of membrane proteins present at the surface of the parasitic protozoan Leishmania. While it harbours leucine rich repeats, which are suggestive of its involvement in parasite-to-host physical interactions, its exact role is largely unknown. Furthermore, the extent of diversity of this gene family, both in copy number and sequence has not been established.
\end{abstract}

Results: From the newly available complete genome sequences of $L$. major, $L$. infantum and $L$. braziliensis, we have established the complete list of PSA genes, based on the conservation of specific domain architecture. The latter includes an array of leucine rich repeats of unique signature flanked by conserved cysteine-rich domains. All PSA genes code either for secreted or membraneanchored surface proteins. Besides the few previously identified PSA genes, which are shown here to be part of a relatively large subclass of PSA genes located on chromosome 12, this study identifies seven other PSA subtypes. The latter, whose genes lie on chromosomes 5, 9, 2I and $3 \mathrm{I}$ in all three species, form single gene (two genes in one instance) subfamilies, which phylogenetically cluster as highly related orthologs. On the other hand, genes found on chromosome 12 generally show high diversification, as reflected in greater sequence divergence between species, and in an extended set of divergent paralogs. Moreover, we show that the latter genes are submitted to strong positive selection. We also provide evidence that evolution of these genes is driven by intra- and intergenic recombination, thereby modulating the number of LRRs in protein and generating chimeric genes.

Conclusion: PSA is a Leishmania family of membrane-bound or secreted proteins, whose main signature consists in a specific LRR sequence. All PSA genes found in the genomes of three sequenced Leishmania species unambiguously distribute into eight subfamilies of orthologs. Seven of these are evolving relatively slowly and could correspond to basic functions related to parasite/ host interactions. On the opposite, the other PSA gene class, which include all so far experimentally studied PSA genes, could be involved in more specialised adaptative functions. 


\section{Background}

Leishmania is a parasitic protozoan of the Trypanosomatidae family and is the pathogenic agent of leishmaniases in humans $[1,2]$. These diseases are endemic in 88 countries on five continents, and cover a wide range of symptoms, from asymptomatic form and benign localised cutaneous lesions to mucocutaneous lesions and visceral outcome. While localised cutaneous lesions can be self-healing, the outcome of the other forms of the disease is more disabling, and the visceral forms are generally fatal if untreated by chemotherapy. The nature of these symptoms appears statistically associated with the parasite species. L. major and L. tropica mainly produce benign cutaneous forms, L. braziliensis, mucocutaneous forms and $L$. infantum and L.donovani generally cause visceral disorders. Leishmania undergoes two major developmental stages in its life cycle: one stage in an invertebrate host, the phlebotomine sand fly and one stage in a vertebrate host (wide range of hosts such as humans, canids, rodents, etc.). In the former, it proliferates in the sandfly gut as a flagellated form, promastigote, and later differentiates into the infectious metacyclic promastigote form while in vertebrate hosts it proliferates intracellularly as an a-flagellated form, amastigotes, after infection of macrophages. The parasite transits from one host to the other when the sandfly feeds by sucking the blood of the vertebrate host.

To understand host-parasite interactions at the molecular level, many studies have focused on the potential role of macromolecules present at the surface of the parasite. These include glycoinositolphospholipids (GIPL), lipophosphoglycan (LPG), as well as the membrane proteins proteophosphoglycan (PPG), MSP/GP63 endopeptidases, PSA (PSA-2/GP46) and amastins. Most of these membrane proteins are glycosylated and anchored via a covalent glycosylphosphatidylinositol (GPI) moiety [3]. Some isoforms of PPG and PSA are also exported outside the parasite as soluble proteins $[4,5]$. LPG plays an important role in binding to the phlebotomine midgut. Furthermore, in conjunction with the MSP proteins, it is involved in the first steps of the vertebrate host infection. They promote resistance to complement, binding to and internalisation in macrophages through the CR3 ligand and fibronectin receptors, and finally inhibition of the oxidative burst $[6,7]$. GIPLs and the cysteine proteases CPB are involved in later stages of infection like protection against the nitric oxide production and modulation of the immune response [8].

PSA proteins have been detected in both promastigote and amastigote stages $[9,10]$. However, the levels drastically increase from exponentially growing to stationary phase parasites, which suggests that PSA are strongly overexpressed in metacyclic promastigotes. This overexpres- sion seems linked with the virulence status of the parasites since serial passaged in vitro cultured promastigotes with low virulence do not show this burst of PSA expression in stationary phase [10]. This expression profile is consistent with the only known role for PSA: resistance to complement lysis [11]. But other roles are possible, either in the late parasite-phlebotomine or metacyclic/amastigotemacrophage interactions. The most suggestive functional determinant in the PSA2 primary structure is the presence of leucine rich repeats (LRR). LRRs are primarily known to be involved in protein-protein and protein-glycolipids interactions. Interestingly, many known LRR containing proteins are involved in host-pathogen interactions. Yersinia invasin, Listeria internalins and Streptococci LrrG protein are surface proteins that play key roles in binding and internalisation of these bacteria in their mammalian hosts $[12,13]$. Likewise, the mammalian Toll-like receptor and Nod families [14], and the plant NBS-LRR resistance proteins [15] are primary actors of the innate immunological system that recognize and bind proteins, glycolipids and nucleic acids of pathogens.

As for the other membrane proteins above, PSAs are encoded by multicopy genes. Up to now, only one or two PSA genes have been described in L. amazonensis [16], $L$. major [9] and L. infantum [17], and before the complete sequencing of $L$. major genome, the number of copies in a single species was largely unknown. Here we have taken advantage of the newly available complete genome sequences of the three species $L$. major, $L$. infantum and $L$. braziliensis $[18,19]$ to draw the precise structure of the PSA gene family. After analysis of the gene phylogeny, which might serve in the identification of distinct PSA functions, we have investigated for the presence of recombination and positive selection as driving forces acting on the evolution of this multigene family.

\section{Results}

\section{The PSA gene family}

A BLAST search analysis was performed against the complete genome sequences of the three species $L$. major, $L$. infantum and L. braziliensis http://www.genedb.org/, using as a query sequence some of the PSA protein sequences reported in the literature. Some genes were discarded from the candidate PSA genes obtained from this BLAST analysis: LmjF12.1005, LinJ12_v4.0662, LbrM_V2.1660 and proteophosphoglycan (PPG) genes (4 in each species). LmjF12.1005 of L. major and LinJ12_v4.0662 of L. infantum, which code for N-terminal truncated forms of PSA (LmjF12.1005 protein actually lacks a LRR domain) are probably pseudogenes. LbrM_V2.1660 is almost identical to gene LbrM_V2.1670, besides harbouring a N-terminal truncation, but this is questionable since the genomic sequence immediately upstream LbrM_V2.1660 is lacking, which renders the determination of the $\mathrm{N}$-terminus 
uncertain. PPGs indeed possess PSA-related LRR motifs, but their overall domain architecture differs from that of PSA proteins. More importantly, PPGs are most probably functionally different from PSA, owing to their very long (often more than 1000 residues) serine-rich stretches on which are anchored very large amounts of phosphoglycan chains [5]. At the end, 32, 14 and 8 PSA genes were found in $L$. major, $L$. infantum and $L$. braziliensis, respectively. Analysis of the primary structure of the encoded PSA proteins reveals that they all possess the same architecture (Fig. 1). The central part of the protein is composed of LRRs in tandem. These repeats, which show high variability, both in terms of number of repeats and primary sequence, are flanked by relatively well conserved domains, including Cys-rich domains, which are expected for extracellular LRR-containing proteins [20]. On the Nterminal side of the LRR domain, one finds a 29 to 31 residue-long domain reminiscent of the LRR motif, but which is clearly not a LRR. This is preceded by a $\mathrm{Cx}(6) \mathrm{C}$ domain and finally by a signal peptide. At the other extremity, the last LRR is followed by a CGCx(2326)CxxxxxC containing domain. This marks the end of the sequence for some PSA proteins. Others have extensions that contain either a Thr/Ser-rich region of variable length or a transmembrane domain preceded by a Cysrich region, or both. This configuration of a N-terminal signal sequence and a C-terminal transmembrane domain predicts that all PSA proteins are either secreted or anchored to the plasma membrane by their C-terminal end. Furthermore, all PSA proteins containing a C-termi- nal transmembrane domain (and a signal peptide) are predicted to be GPI-anchored (see Fig. 2).

The main signature of PSA proteins is their specific 24-25 amino-acids LRR motif, of consensus sequence $\mathrm{G}(\mathrm{T} /$ S)LPxxWxx (M/I)xxLxxLxLxxxx(x)(V/L/I)(S/T) (Fig. 1). The most striking feature of this motif relative to other LRRs is the presence of quasi invariant glycine, proline and tryptophane residues at positions 1, 4 and 7, respectively. This motif is not present in the predicted proteins from the fully sequenced genomes of two other trypanosomatidae, T. brucei and T. cruzi, and no clear homologs of PSA could be identified in these organisms. The only PSA domain for which homology was found in the other trypanosomatidae is the Thr/Ser-rich domain. The T(n)KP2 and T(n)EAPT repeats found in most of these domains are present in $T$. cruzi mucins. However, while it makes up the bulk of the mucin protein architecture, this domain is not always present in PSA proteins (see Fig. 2), and mucins do not contain any LRR repeat. Therefore, PSA proteins and mucins are likely to play different roles. Actually, the LRRs mostly similar to those of Leishmania PSA proteins belong to the plant NBS-LRR resistance proteins.

PSA genes are dispersed on chromosomes (Chr) 5, 9, 12, 21 and 31 for all three Leishmania species. Chromosomes 9 and 21 contain a single gene, while Chr 5 contains 2 genes, and Chr 31, 3 genes ( 4 in L. major). PSA genes localised on different chromosomes (and also those of the dif-
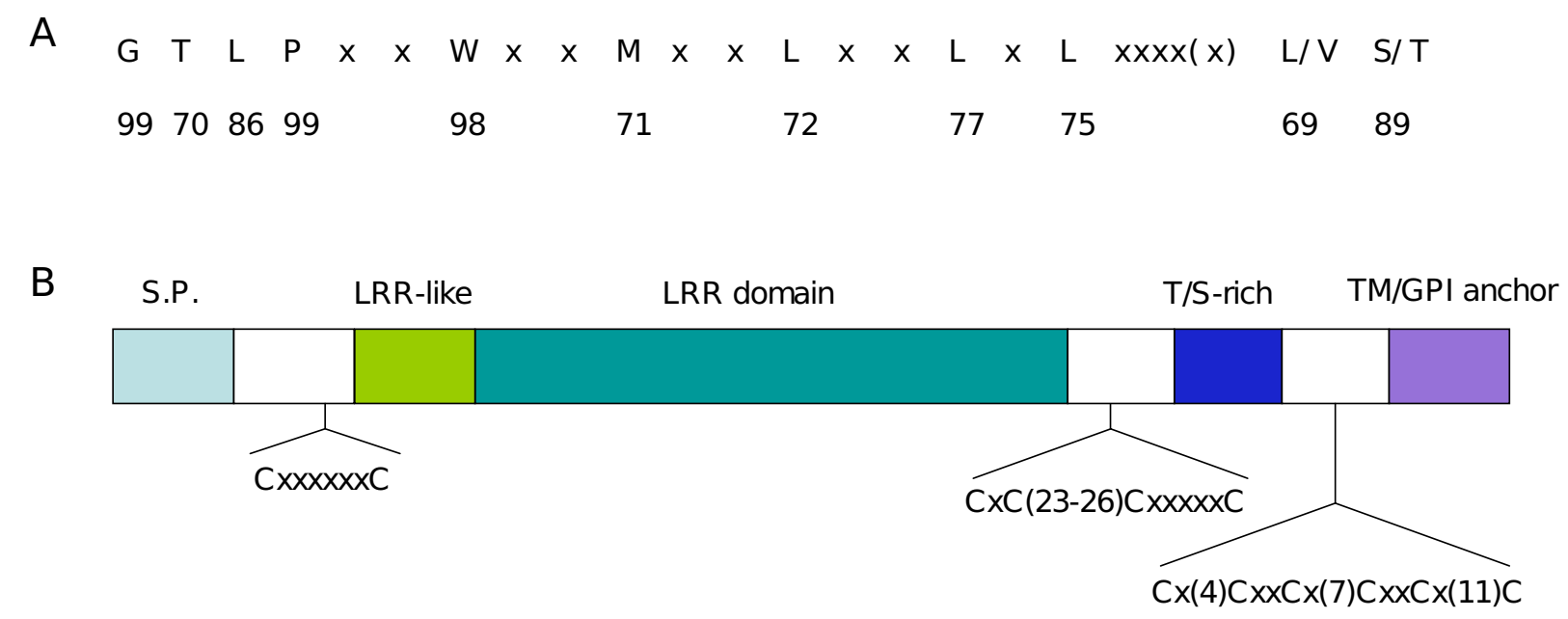

\section{Figure I}

Analysis of the primary structure of PSA proteins. A) Consensus sequence of the PSA LRR repeats. Numbers indicate the percentage of occurrence in the LRR repeats of the corresponding residues above. Residues in position \#3, 7, I0, I3, 16, 18, 23(or 24) are always hydrophobic. B) Schematic representation of the PSA domain architecture. S.P: signal peptide; T/S: threonine/serine TM: transmembrane domain. 


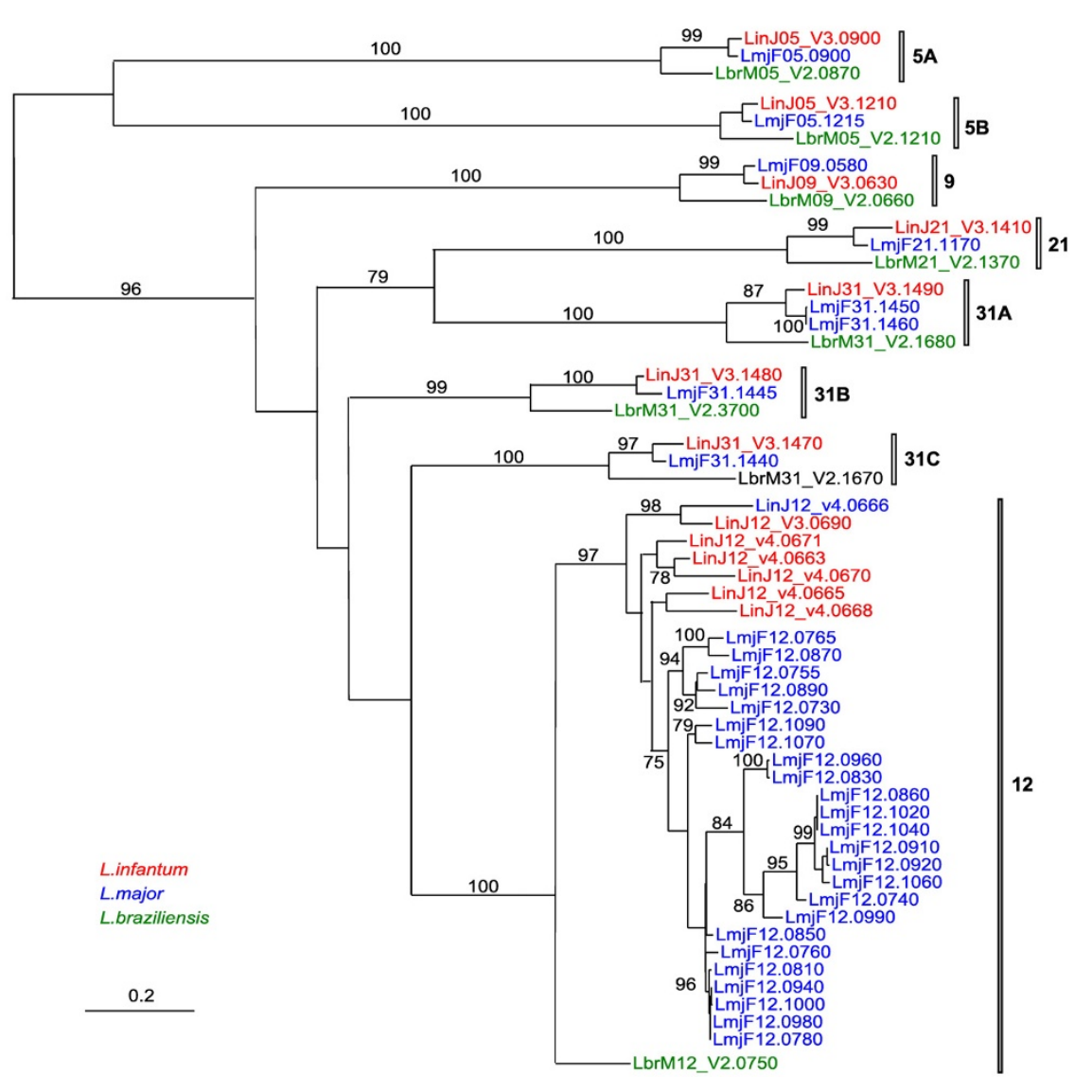

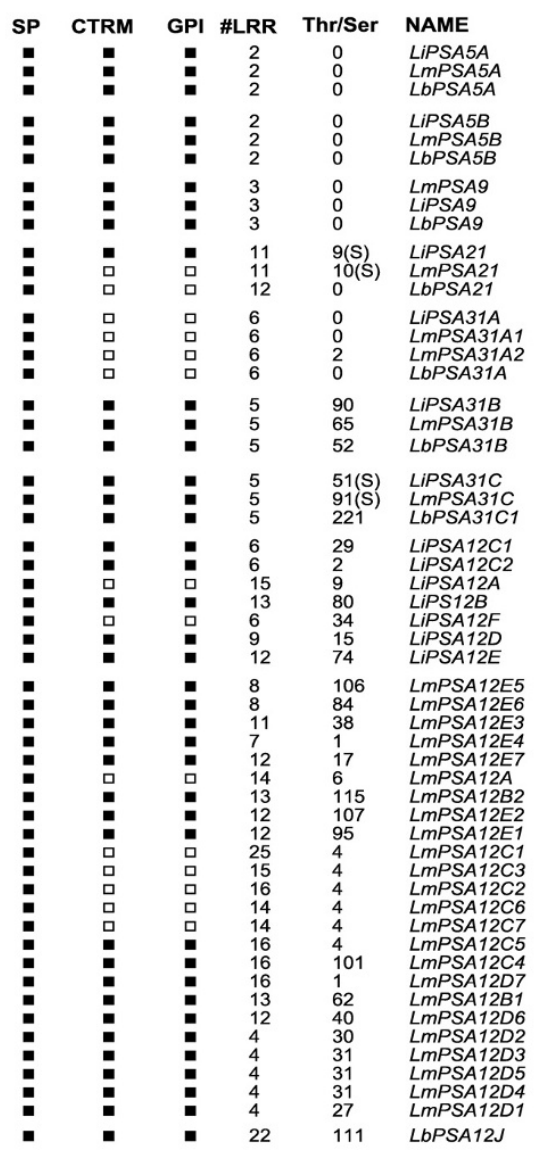

Figure 2

Phylogenetic analysis of the PSA gene family. A phylogenetic tree was inferred by PhyML for a DNA alignment of the PSA ORFs of $L$. braziliensis, $L$. major and $L$. infantum, excluding the Thr/Ser and Cys-rich/transmembrane C-terminal domains as well as all but the C-ter most LRR repeat (see Additional file I). Only bootstrap values (from 500 replicates) greater than $74 \%$ are indicated. Very similar trees (with same significant clustering of genes) were obtained with DNA or protein sequence alignments, using both maximum likelihood or distance methods (not shown). SP: signal peptide; \#LRR: number of LRR repeats (number in brackets refers to additional LRRs that diverge from the consensus LRR sequence). T/S: number of Thr and Ser residues in the Thr-rich domain (or Ser-rich, indicated by (S)). CRTM: cysteine-rich and C-ter transmembrane domains. GPI: GPI anchor. Filled and open squares indicate the presence or absence of the above features, respectively.

ferent Chr31 loci) are sufficiently divergent to define PSA gene classes (see below). Inside each chromosome, all PSA genes are clustered in tandem, except those of Chr 5 and gene LbrM31_V2.3700, which are well separated. The PSA subfamily present on Chr 12 is the most complex (except in L. braziliensis where there is only one gene). In L. major, the $24 \mathrm{Chr} 12$ PSA genes belong to a single cluster of tandem repeats, with many of these genes separated by a single non-related intervening gene. This pattern is also observed for the 7 Chr12 PSA genes of L. infantum. All PSA genes arranged in clusters have the same orientation relative to transcription. The only PSA genes studied so far in vivo belong to the Chr12 array. Although the PSA genes we have identified on the other chromosomes show significant sequence divergence with the Chr12 genes (see below), the conservation of the precise domain organisa- tion between all these genes justifies their being included in the same unique PSA family.

\section{Phylogeny of the PSA gene family}

An alignment of all PSA protein sequences from all three Leishmania species was generated. For phylogenic analysis, only the domains present in all PSA proteins were included in the alignment. The Thr/Ser and Cys-rich/ transmembrane C-terminal domains were thus discarded, as well as the central LRR domain (except for the last LRR motif of each sequence, which was retained). LRR units probably evolve not only by nucleotide substitution but also by birth and loss of repeats following recombination events. Therefore, alignments of rather distant LRR domains (with low similarity between repeats and varying number of repeats) may not reflect properly their evolu- 
tion. This truncated alignment was transformed into its DNA coding version (Additional file 1), which was used to generate a phylogenetic tree by PhyML (Fig. 2). This tree shows the existence of eight PSA subfamilies which can be defined by their chromosome localisation: Chr5 (A and B), 9, 12, 21 and three subfamilies on Chr31 (A, B, C). Subfamilies Chr5A and B are the most distantly related and pair apart from all other clusters. Among the latter, only the Chr21/Chr31A subfamilies can be phylogenetically linked with high confidence bootstrap values. Each of the three Leishmania species has at least one gene in each of these eight subfamilies. The L. infantum gene orthologs always group with those of L. major, which is expected from the known species tree. The relatively short branch length separating orthologs in comparison to those separating the inparalogs (ancestors of the seven gene subfamilies) might suggest that speciation of the three Leishmania is a late event in the evolution history of the PSA genes. In keeping with this interpretation, a tree with similar relative branch lengths was inferred when using the rate of synonymous substitutions $\left(d_{\mathrm{S}}\right)$, a more neutral distance metric (not shown).

\section{Chr5A, B, 9, 2I, 3 I A, B, C subfamilies of PSA genes}

While the Chr12 subfamily is most diversified, having very different numbers ( 1 to 24 ) of rather distantly related paralogs in the three species, the PSA genes lying on the other chromosomes form very simple clusters. For each of the latter subfamilies, each species contains a single gene (except $L$. major, which has two highly similar paralogs for the Chr31A cluster) and the orthologs or pseudoorthologs are quite similar (median $=75 \%$ amino acid identity for all three species and $93 \%$ for $L$. infantum/L. major orthologs). This degree of homology between orthologs, which was calculated on the same domains used above for phylogenetic tree determination, was also observed over the entire LRR domain. Indeed, confident alignments covering this domain can be obtained inside each of the Chr5A and B, 9, 21, 31A, B, and C clusters since the number of LRRs differ at most by one inside each subfamily and the similarity between orthologs is (median $=80 \%$ amino acid identity for all three species and $96 \%$ for L. infantum/L. major orthologs) (see Additional files 2, 3, 4, 5, 6, 7, 8 for sequence alignments). On the other hand, similarity between genes of different subfamilies is rather low over this domain (median $=32 \%$ amino acid identity, and much difference in number of repeats). This may suggest that the LRR domains characteristic of each cluster represent specific binding properties, which could confer specialized roles to their respective PSA proteins. Another link between these subfamilies and functional specialisation is given by the nature of the C-terminal domain. Indeed, all members of the same subfamily share the same membrane anchorage/ secretion determinant. In particular, all genes of the phyl- ogenetically linked Chr21 and 31A subgroups are predicted to code for secreted proteins (Fig. 2).

\section{Chrl 2 subfamily of PSA genes}

The tree of Fig. 2 shows that the phylogenetic structure of the Chr12 genes is quite different from that of the above subfamilies. Here, all genes group according to the species, which suggests that generation of paralogs in L. major and L. infantum occurred independently in each species. This clustering pattern was also obtained for an alignment corresponding to the C-ter extension containing the Cysrich/transmembrane domains of the relevant subset of Chr12 PSA genes (not shown). We then produced an alignment of the LRR domain for these Chr12 genes. Although sequence diversity and variability in numbers of repeats render this task uncertain, this alignment could be validated by the high similarity between many genes and singularities of some LRRs (length of LRRs, sequence specificities). The most robust portion of this LRR alignment (Additional file 9) was used to generate a phylogenetic tree (Fig. 3). While many clades were conserved from the tree of Fig. 2, new well supported clusters were generated. Notably, two link together genes from $L$. major and $L$. infantum, thus identifying clear orthologs: LmjF12.1090/ LinJ12_v4.0671 and LmjF12.0850, LmjF12.1070/ LinJ12_v4.0663. Phylogenetic analysis scanning of all individual domains including single LRR repeats confirmed the above orthologies for all LRR repeats except those at both ends of the LRR domain (not shown). On the other hand, the $\mathrm{N}$ - and C-terminal domains, particularly the signal peptide region and the Cys-rich/transmembrane domain are species specific. The identification of LmjF12.1090/LinJ12_v4.0671 as orthologous genes was also strengthened by the absence of C-terminal Cysrich and transmembrane coding domains in their respective ORFs. Yet, these domains are still encoded by very similar sequences located immediately downstream of the stop codons, suggesting that a common frameshift mutation resulted in this deletion. Besides the above orthologs, some clustering of $L$. infantum with $L$. major genes was observed, but with low bootstrap values. New trees were generated for the L. infantum genes alone, or for those of L. major, but they did not reveal any new well-supported clusters. If we consider the LRR domain as the main functional determinant of the PSA protein, the following picture of the genes on Chr12 emerges. In L. infantum, only two genes (LinJ12_v4.0666 and LinJ12_V3.0690) are closely related. Besides this clade, the history of the $L$. infantum Chr12 genes can not be resolved, due to the important apparent divergence between the sequences, which might result in part of recombination events involving LRR repeats. In L. major, we can distinguish four clusters of paralogs. Only three of them are supported by strong bootstrap values. Cluster Lm12B contains the two pseudo-orthologs of LinJ12_v4.0663. Subgroup C com- 


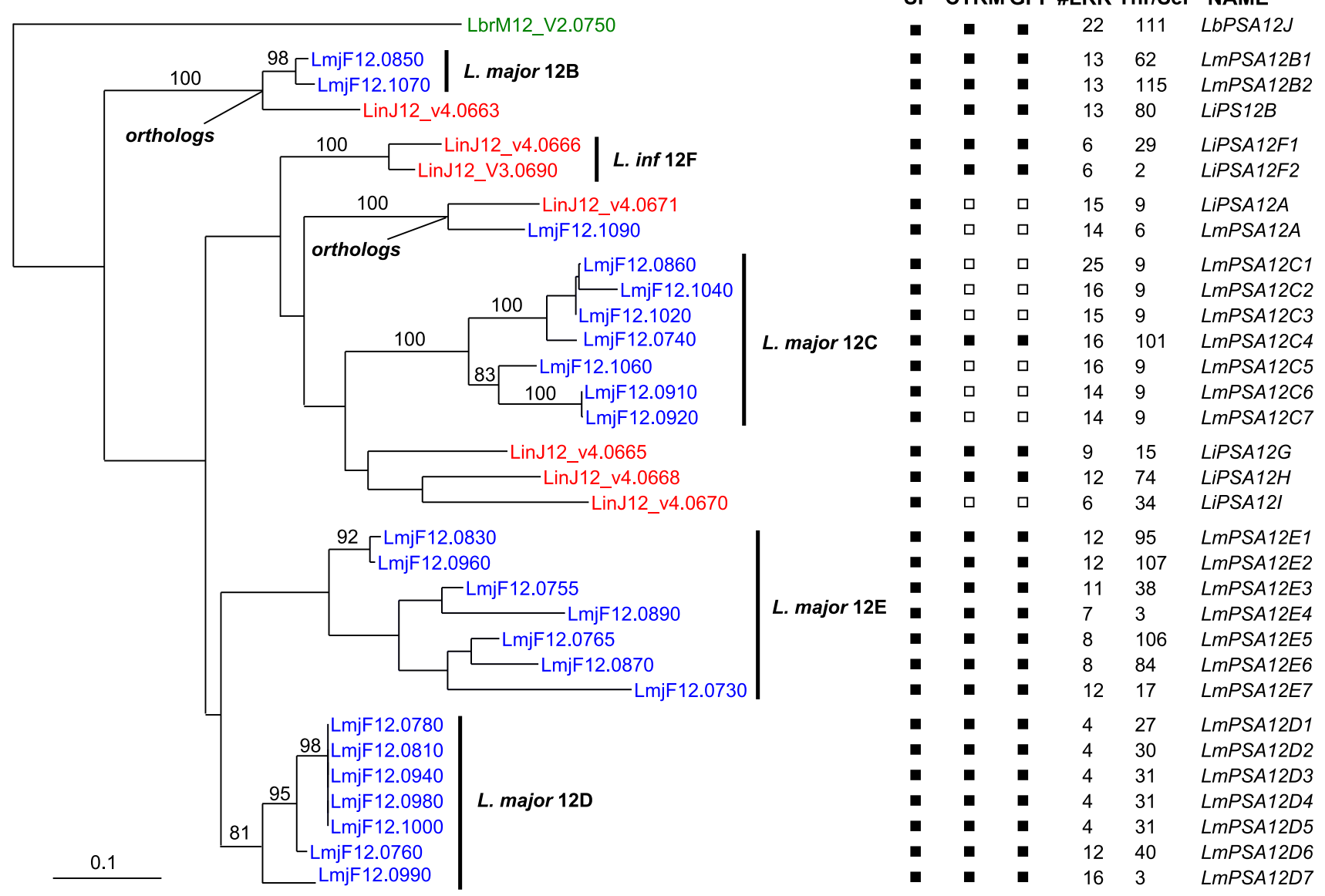

Figure 3

Phylogenetic tree of the LRR domains of PSA genes located on chromosome I 2. A phylogenetic tree was inferred by PhyML for a DNA alignment of the LRR domain (see Additional files 9 and I 3 for protein and DNA alignments, respectively) of all Chr 12 PSA genes of $L$. braziliensis, L.major and $L$. infantum. Bootstrap values (from 500 replicates) greater than $74 \%$ are indicated. Very similar trees (with same significant clustering of genes) were obtained with DNA or protein sequence alignments and by maximum likelihood or distance methods (not shown). SP: signal peptide; \#LRR: number of LRR repeats. T/S: number of Thr and Ser residues in the Thr-rich domain (or Ser-rich, indicated by (S)). CRTM: cysteine-rich and C-ter transmembrane domains. GPI: GPI anchor. Filled and open squares indicate the presence or absence of the above features, respectively.

prises seven genes, out of which six code for secreted proteins. As for the LmjF12.1090/LinJ12_v4.0671 genes, truncation of the Cys-rich and transmembrane domains in these genes originated from a common non-sense frameshift mutation. Cluster D comprises seven genes, five of which are almost identical (>99\% nucleotide identity) and were most probably created by recent duplication events. In comparison to L. infantum, L. major has a much younger cluster of Chr12 genes, with 9 recently duplicated genes $\left(d_{\mathrm{S}}<0.02\right.$ and no insertions or deletions). We must note, however, that nearly identical copies of PSA genes might have been missed in the $L$. infantum genome assembly, where contigs were assembled solely from shotgun sequence reads.

\section{Recombination in PSA genes}

The repetitive nature of the LRR domain and the number of paralogs imply that recombination has largely contributed to the diversity of the PSA gene. Some recombination events can be traced in the LRR domains. For example, while LRRs inside a given PSA protein are generally quite divergent, nearly identical repeats can be found. For example, LbrM12_V2.0750 has tandem triplicates of a LRR doublet, and LmjF12.0860, 1020, 1040 have up to 19 
copies of a conserved LRR motif. These nearly exact duplications could have been generated by recent intragenic cross-overs, or cross-overs involving closely related paralogs. More diverging chimeric genes could result from intergenic recombination. To detect such events, we partitioned the LRR alignment of the L. major Chr12 genes into single LRR alignments and inferred the phylogenetic tree for the most conserved of them. Fig. 4 shows how these genes cluster according to these LRRs as well as to the conserved regions outside the LRR domain. It can be noticed that while genes from clade $\mathrm{C}$ are quite homogeneous in their clustering pattern across the entire alignment, others are more mosaic. This is the case for most of the genes of clade $\mathrm{E}$, and this probably explains the low resolution (weak bootstrap values) of this subtree (see Fig. 3). Simple intergenic recombination events are readily suggested. For example, genes LmjF12.0830 and 12.0960 appear to be chimeric products originating from strand exchanges between genes belonging to clades $\mathrm{D}$ and $\mathrm{E}$. Likewise, gene LmjF12.0990 must have been generated by the fusion of the N-terminal pre-LRR domain of a gene from clade $\mathrm{C}$ with a gene of clade $\mathrm{D}$. To validate these conclusions, alignments of relevant PSA gene domains were analysed by SimPlot (Fig. 5) [21]. First, similarity profiles against the putative chimeric genes (LmjF12.0830, LmjF12.0960 and LmjF12.0990) show a clear switch between potential parent genes at the breakpoint region suggested above in Fig. 4. Second, bootscanning analysis reveals an alternation of high bootstrap support across the same regions. Finally, these recombination events are confirmed by analysis of informative sites by the Maximum Chi-Squared method (Fig. 5).

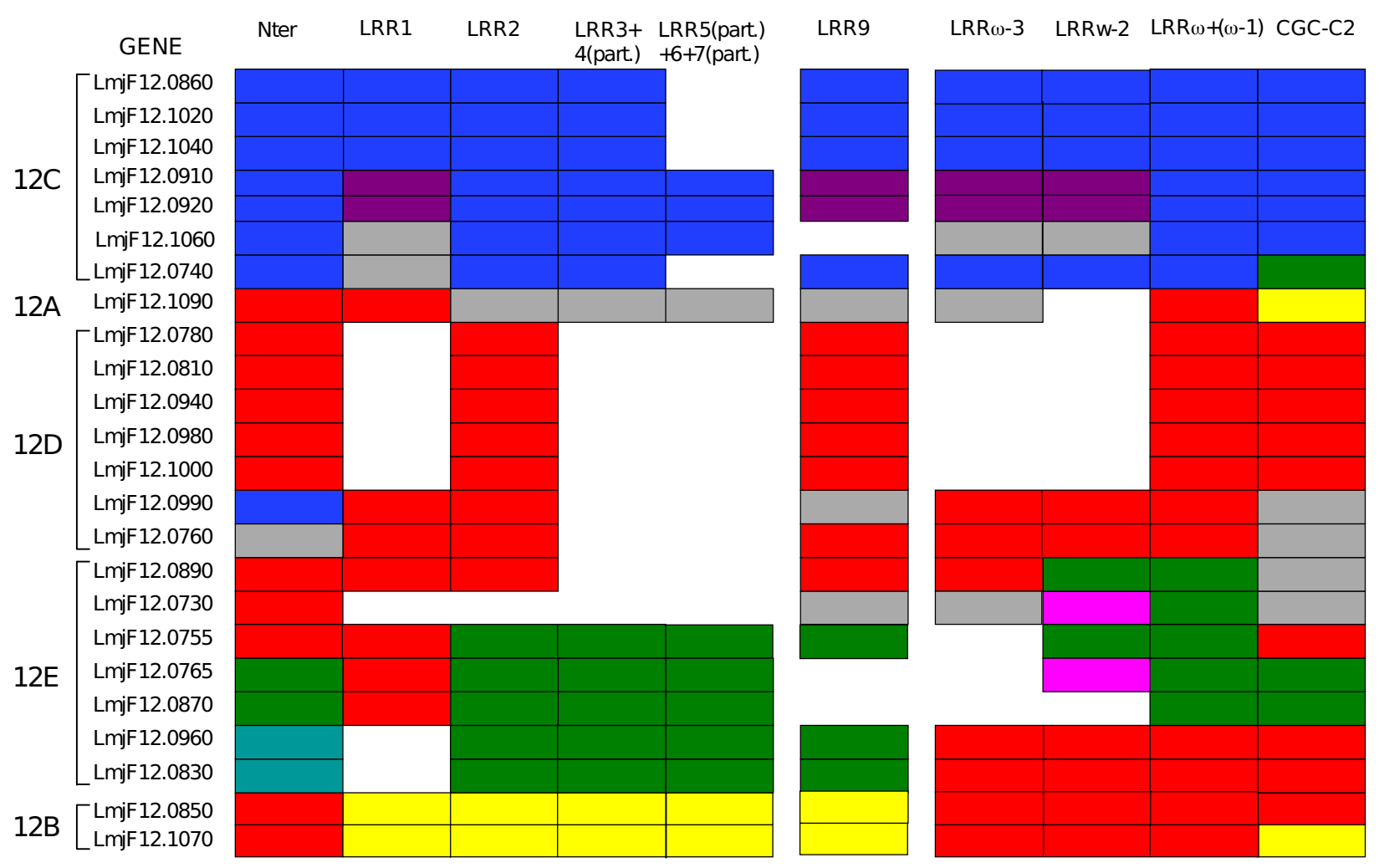

\section{Figure 4}

Phylogenetic clustering of individual PSA domains. Trees were inferred by Neighbor-Joining (NJ) from nucleotide distance matrix (TN93 model) calculated individually for all the indicated domains of the L. major Chr I 2 PSA genes. Individual LRR domains that were present in only a few genes were not analysed. For each domain, those genes that cluster together in the tree (with bootstrap values greater than 74\%) are boxed with the same colour. Grey boxes indicate genes that do not cluster with any other genes. Absence of a box indicates that the corresponding domain is absent in the gene. N-ter: PSA N-terminal domain up to the first LRR repeat. LRRs are numbered from $\mathrm{N}$-ter to $\mathrm{C}$-ter according to the LRR alignment (see Additional file 9). $\omega$ : refers to the last (C-ter most) LRR of each gene; therefore, $L R R \omega+(\omega-I)$ corresponds to the sequence comprising the last and penultimate LRRs in each gene. (part.): partial; for example, 4 (part.) means portion of LRR 4. CGC-C2: the CGCx(2326) $\mathrm{Cx} x \mathrm{xx} \mathrm{C}$ domain at the C-ter end of the LRR domain. 
A) $\operatorname{LmjF} 12.0990$

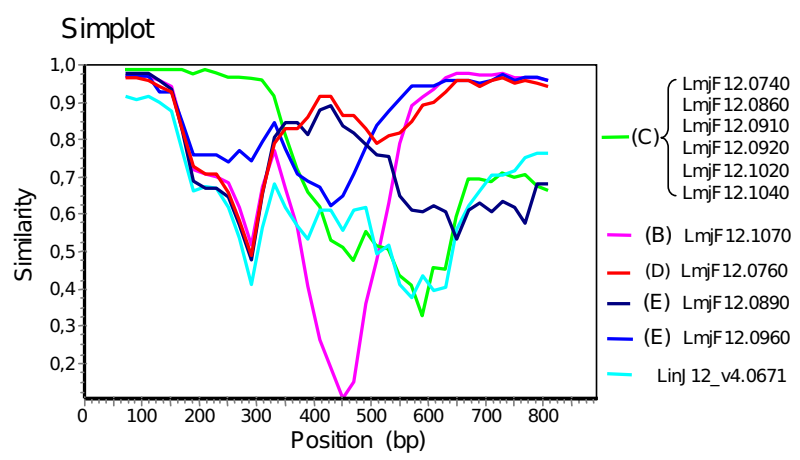

Bootscan

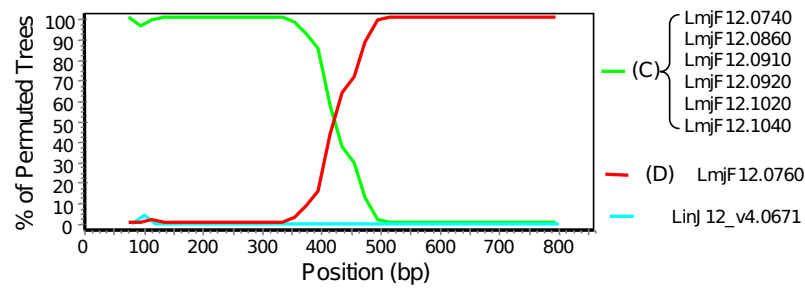

MaxChi2

\begin{tabular}{|c|c|c|}
\hline \multirow{2}{*}{$\begin{array}{l}\text { Bifurcating } \\
\text { tree }\end{array}$} & \multicolumn{2}{|c|}{$\mathrm{N}$ (informative sites) } \\
\hline & $<\# 364$ & $>\# 364$ \\
\hline${ }_{c}^{990} \succ_{671}^{760}$ & 34 & 1 \\
\hline $\begin{array}{l}990 \\
760\end{array}{ }_{671}^{\mathrm{C}}$ & 0 & 41 \\
\hline $\begin{array}{l}990 \\
671\end{array}{ }_{760}^{\mathrm{C}}$ & 0 & 1 \\
\hline
\end{tabular}

B) LmjF 12.0960
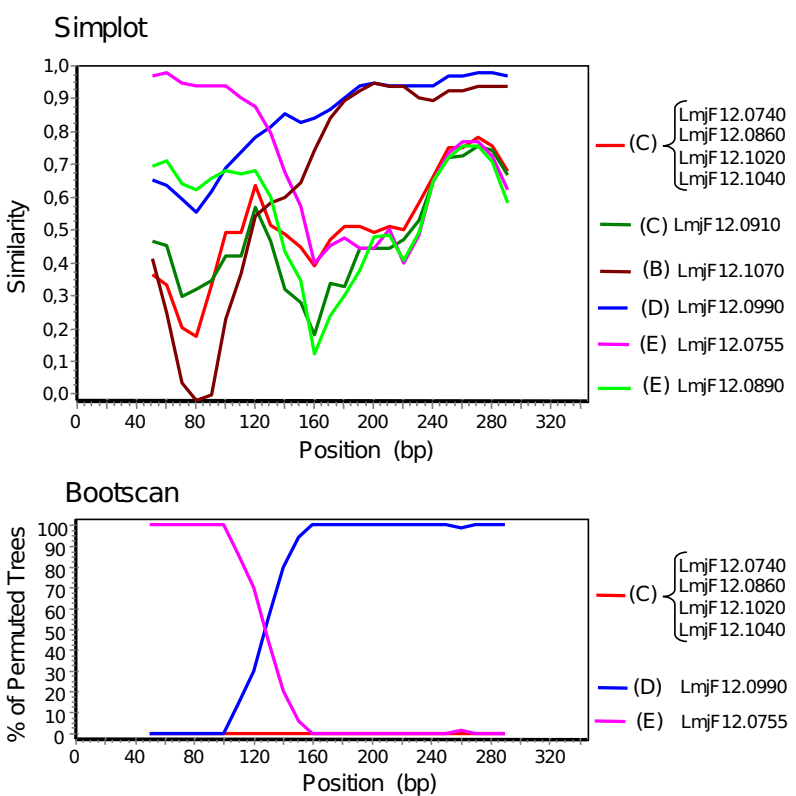

MaxChi2

\begin{tabular}{|c|c|c|}
\hline Bifurcating & N. (infor & ve sites) \\
\hline tree & $<\# 150$ & $>\# 150$ \\
\hline $\begin{array}{l}960 \\
755\end{array}{ }_{C}^{990}$ & 11 & 0 \\
\hline${ }_{990}^{960} \varkappa_{C}^{755}$ & 0 & 26 \\
\hline${ }_{\mathrm{C}}^{960} \succ_{990}^{755}$ & 0 & 0 \\
\hline
\end{tabular}

\section{Figure 5}

Analysis of chimeric PSA genes by Simplot. A) LmjFI2.0990. A DNA alignment of the indicated genes covering LRR \#2, 9 and the last $4 \mathrm{C}$-ter most LRRs, together with the N-ter and CGC-C2 flanking domains (Additional file 14) was analysed with Simplot. Single or multiple genes from each phylogenetic PSA gene cluster of L. major Chr I 2 (see Fig. 3) were first used for similarity analysis (Simplot) against the LmjFI2.0990 putative chimeric gene. The six genes of cluster I2C were reduced to a consensus sequence. The best pair of candidate parental genes (LmjFI2.0760 and consensus sequence for cluster I2C) and a control gene were then used for bootscanning and informative sites analysis (MaxChi2). B) LmjFI2.0960. The same analysis was performed as in A) using a DNA alignment covering LRR \#2, 9 and the last 3 C-ter most LRRs (see Additional file I5).

More profound rearrangements can be created by intergenic recombinations (both at coding and non-coding sites), which lead to whole gene duplication and deletions. One such rearrangement can be traced in the $L$. major Chr1 2 PSA gene cluster, where PSA genes are organised in tandem, tough often separated by one of two unrelated genes of type A or $\mathrm{B}$. The distribution of these intervening genes together with the phylogenic signature of each PSA genes suggest that the two pairs of very similar paralogs LmjF12.0810/LmjF12.0940 and LmjF12.0830/ LmjF12.0960 were created by a same segmental duplication involving a bloc of five genes (see Additional file 10).

\section{Positive selection of PSA genes}

The high gene diversity found for the Chr12 subfamily in $L$. major and $L$. infantum could be indicative of selective pressure acting on these genes. The preservation of a high number of paralogs on chromosome 12 (with the exception of the single L. braziliensis Chr12 gene) in comparison to that of paralogs found on the other chromosomes (one or two copies only) is itself an indication that diversification is taking place in the Chr12 subfamily. This is strengthened by the high protein sequence diversity among the L. infantum and L. major paralogs of Chr 12 . After grouping pairs of genes with $d_{\mathrm{S}}$ values below 0.02 (recently duplicated genes) as single entities, we found that the median pairwise $p$-distances (calculated over the coding sequence deleted of the Thr/Ser and Cys-rich/ 
transmembrane C-terminal domains) for the L. infantum and L. major Chr12 paralogs were 0.336 and 0.285 , respectively (it was only slightly higher, 0.348 , for the $L$. infantum-L. major genes pairwise comparison). That is to compare to the high protein similarity found between the L. infantum and L. major orthologs of Chr5, 9, 21, 31, ranging from 91 to $95 \%$ identity (median $=94 \%$ ). Orthologs of PSA genes on Chr5, 9, 21, 31 are therefore much more conserved even than paralogs of Chr12. To gain a better insight on selective pressure, we conducted analysis of ratios of $d_{S}$, the number of synonymous substitutions per synonymous sites, to $d_{N}$, the number of non-synonymous substitutions per non-synonymous sites, over alignments covering the entire LRR domain. We calculated a median $d_{\mathrm{N}} / d_{\mathrm{S}}$ ratio of 0.26 for the pairwise comparison of Chr 5,9 , 21,31 orthologs of $L$. infantum and L. major ( 0.26 when those of $L$. braziliensis where included). The pairwise comparison between all genes of Chr12 of L. infantum and $L$. major (after grouping pairs of genes with $d_{\mathrm{S}}$ values below 0.02 as single entities) revealed a much higher median $d_{\mathrm{N}} /$ $d_{\mathrm{S}}$ of 0.74 ( 0.71 and 0.86 when comparing only paralogs of $L$. major and L. infantum, respectively). Fig. 6 shows the $d_{\mathrm{N}} / d_{\mathrm{S}}$ versus $d_{\mathrm{S}}$ plot of all pairwise comparisons. $d_{\mathrm{N}} / d_{\mathrm{S}}$ values of the Chr5,9,21,31 orthologs are quite uniformly distributed along the $d_{\mathrm{S}}$ axis, well below those of the Chr12 genes, with the exception of two points which refer to the same LbrM31_V2.1680 gene. It is worth noting that the $d_{\mathrm{N}} / d_{\mathrm{S}}$ values associated with the lowest $d_{\mathrm{S}}$ values for the Chr12 paralogs (and where the alignments are the most confident) are relatively high. The two ortholog pairs of Chr12 genes (LmjF12.1090/LinJ12_v4.0671 and

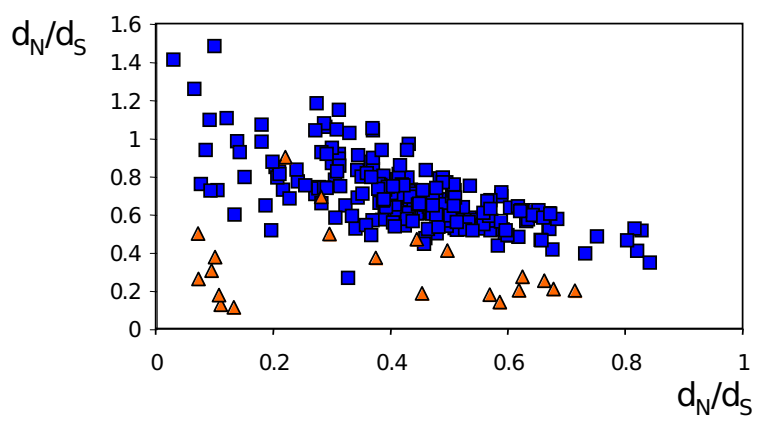

\section{Figure 6}

$d_{N} / d_{S}$ vs $d_{S}$ plots for PSA genes pairwise comparisons. DNA alignments covering the LRR domain were generated for each of the Chr5A, B, 9, I2, 2I, 3IA, B, C PSA gene clusters (including genes of all three Leishmania species; see Additional file $\mathrm{I} 3$ for alignment of Chr I 2 genes). After reducing very homologous genes (with $d_{S}<0.02$ ) to single entities, $d_{N} /$ $d_{S}$ and $d_{S}$ values were calculated for all pairwise comparions inside a given cluster. Orange triangles: Chr5A, B, 9, 2I, 3I A, $B, C$ clusters; blue squares: Chrl 2 cluster. Arrows point to values for $L$. major-L. infantum ortholog comparisons.
LmjF12.0850， LmjF12.1070/LinJ12_v4.0663) position somewhat outside the distribution of Chr12 genes towards low $d_{\mathrm{N}} / d_{\mathrm{S}}$ values, especially for LmjF12.1090/ LinJ12_v4.0671, whose $d_{\mathrm{N}} / d_{\mathrm{S}}$ value $(0.31)$ rather falls inside the Chr5,9,21,31 ortholog values. These results suggest that the PSA genes on chromosome 5, 9, 21 and 31 , as well as orthologs LmjF12.1090/LinJ12_v4.0671 and LmjF12.0850, LmjF12.1070/LinJ12_v4.0663 of chromosome 12 have been more subjected to purifying selection than the other Chr12 genes.

A more incisive method to detect positive selection involves analysing $d_{\mathrm{N}} / d_{\mathrm{S}}$ ratios at each codon site. Indeed, $d_{\mathrm{N}} / d_{\mathrm{S}}$ ratios calculated over the entire length of the sequence cannot always identify positive selection $\left(d_{\mathrm{N}} / d_{\mathrm{S}}\right.$ $>1$ ). That is because only a subset of sites in a given gene is subjected to diversifying selection. The alignment of the entire coding sequence of the Chr12 genes, except the Cterminal Cys-rich and transmembrane domains was analysed using codeml of the PAML package. Likelihood ratio test analysis of three pairs of simple and more complex models of $d_{\mathrm{N}} / d_{\mathrm{S}}$ categories (M1a/M2a, M7/M8 and M8a/ M8) all revealed the presence of positive selection with high probabilities $(P<0.001)$. The M1a/M2a models comparison predicted the presence of 43 positively selected sites with $P<0.05$ (59 sites with $P<0.1$ ), among which 33 (44 with $P<0.1$ ) were located in the LRR domain. Very similar results were obtained with the M7/ M8 and M8a/M8 model comparisons. Codeml also revealed the presence of positive selection $(P<0.001)$ in alignments comprising all Chr 12 genes of either L. infantum or $L$. major alone, or the 7 closely related genes of $L$. major 12B cluster (see tree of Fig. 3). On the other hand, PSA genes on the other chromosomes could not be analysed properly by codeml: each of these phylogenetically defined clusters possess too few genes ( 2 to 4 ), and those often offer poor diversity. It is known that inference of positive selection by codeml can lead to false positives when the genes under investigation have a recombination history [22]. To minimise the impact of recombination, we performed codeml analysis on single or contiguous pairs of LRRs of the Chr12 genes. The majority of these analyses revealed the presence of positive selection and could identify sites under positive selection. Under model M8, still 15 such sites were found at $\mathrm{P}<0.05$ for pairs of LRRs. We then asked if the numerous predicted positively selected sites found above by codeml for the entire LRR domain of Chr12 genes corresponded to particular positions in each LRR. Fig. 7A shows that all these sites are located at non-conserved sites of the LRR consensus 
sequence. The posterior $d_{\mathrm{N}} / d_{\mathrm{S}}$ values calculated at each site by codeml were grouped according to their corresponding amino acid position in the LRR and a mean $d_{\mathrm{N}} / d_{\mathrm{S}}$ value was determined for each residue position in the LRR (Fig. $7 \mathrm{~B})$. As expected, variable positions have higher $d_{\mathrm{N}} / d_{\mathrm{S}}$ values than more conserved positions. An even better correlation is observed if we consider the localisation of the sites in the $3 \mathrm{D}$ structure of the LRRs. Crystal structures and computer modelling of several LRR domains in other proteins allow for the prediction of the positioning of residues outside or inside the LRR domain 3D structure [23]. The relatively well conserved hydrophobic residues are involved in structuring of the consecutive $\alpha$-helices and $\tilde{\beta}$-sheet domains of the LRRs that arrange into a horseshoe shape, and are buried inside the structure. On the opposite, the most divergent residues of the LRR orientate
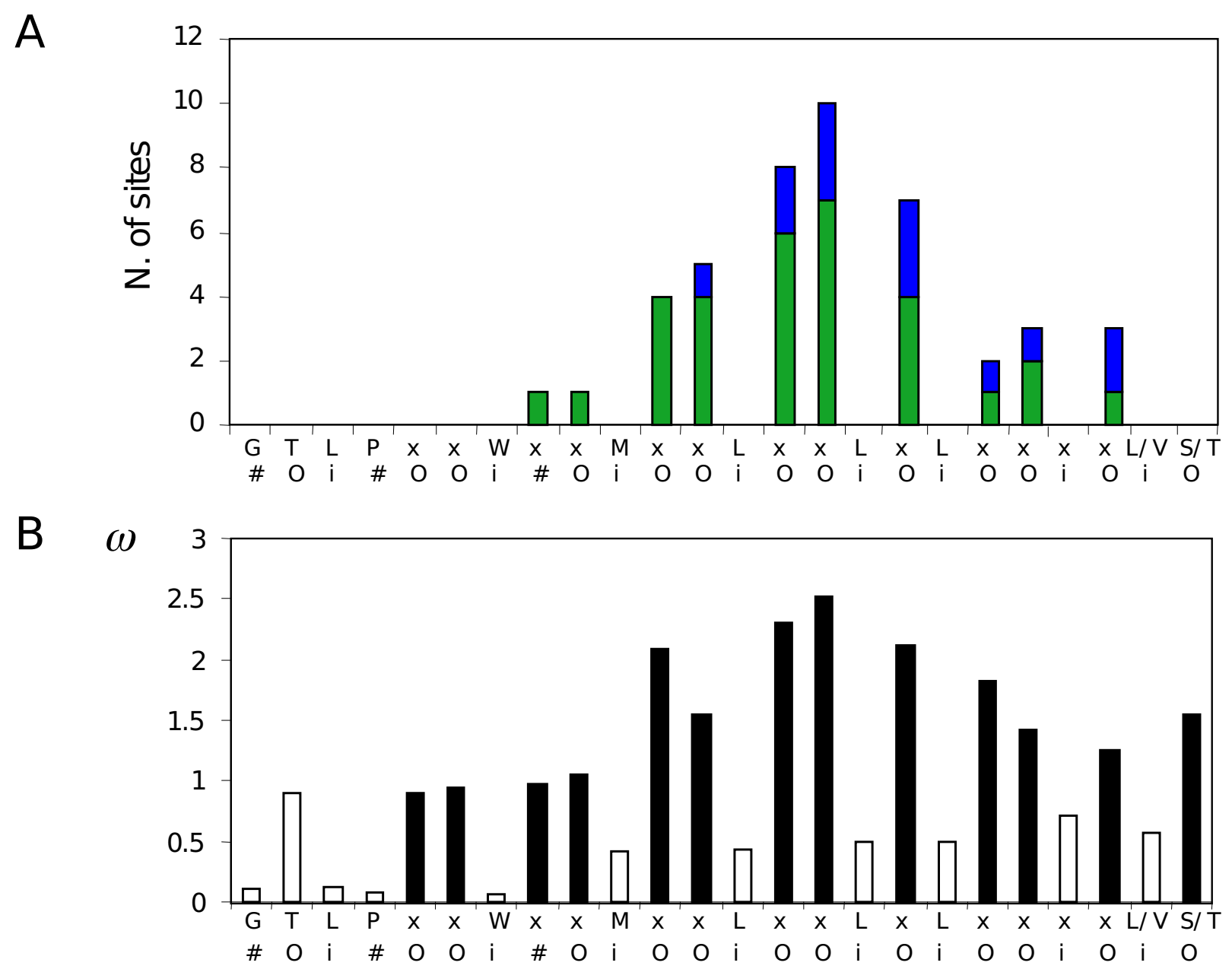

\section{Figure 7}

Distribution of the predicted positively selected sites along the LRR amino acid consensus sequence. A) A DNA alignment covering the LRR domain plus the flanking N-ter and CGC-C2 domains of all Chr I 2 PSA genes (Additional file I6) was analysed by codeml. A) The predicted positively selected sites were plotted against their positioning in the LRR domain. Green bars represent the number of predicted positive sites with probabilities greater than $95 \%$ and blue bars, eventual increments when considering sites with probabilities greater than $90 \%$. o, i and \# refer to positions predicted to reside outside, inside or "in between" in the PSA LRR 3D structure. B) Means of $d_{N} / d_{S}(\omega)$ at each residue position in the LRR were calculated from the posterior $d_{N} / d_{S}$ values of all sites sharing the same position in the LRR. Filled bars: variable residues; open bars: conserved residues. 
outward. It is clear from Fig. 7A that positively selected sites all correspond to positions predicted to reside at the surface of the LRR domain structure. In addition, the latter positions have higher posterior mean values of $d_{\mathrm{N}} / d_{\mathrm{S}}$ (Fig. 7B).

\section{3' Intergenic region}

Expression of some PSA genes of Chr12 has been shown to be stage specific. Strong increases in the levels of both mRNA and protein were observed during metacyclogenesis in vitro [10]. This regulation was shown to depend on the 3' intergenic region (IR) of the PSA gene [17]. The question arises, then, as to whether the different classes of PSA might be regulated the same way. We therefore wished to compare the phylogeny of the 3' (IR) to that obtained above for the coding sequence. Around $2 \mathrm{~kb}$ of the 3' IR of PSA genes from all three Leishmania species (with few exceptions for $L$. infantum and $L$. braziliensis due to gaps in their sequences) were analysed by local BLAST and aligned by Dialign2. We found that the 3' IRs are conserved between all orthologs (and pseudo-orthologs for he Chr12 clade), but show no significant homology between genes of the 8 different PSA clades $(05,09,12$, $21,31 \mathrm{~A}, \mathrm{~B}, \mathrm{C})$. While the similarities found for pairwise comparisons involving $L$. braziliensis sequences are variable and relatively low, those of the other comparisons are high. The L. infantum/L. major orthologs of clades 5, 9, 21, $31 \mathrm{~A}, \mathrm{~B}, \mathrm{C}$ are equally well conserved (ranging from 87 to 92\% identity; see Additional file 11 for sequence alignments), as are the set of pseudo-orthologs and paralogs of clade 12 (median $=95 \%$ identity; see Additional file 12 for sequence alignment). Yet, the alignment of the 3' IR of these Chr12 PSA genes is quite mosaic, showing large blocks of deletions/insertions. Phylogenetic analysis of an alignment retaining $1.2 \mathrm{~kb}$ of domains conserved in at least $70 \%$ of the genes revealed that $\mathrm{Chr} 12$ genes cluster according to the species (not shown). This is reminiscent of the extremities of the coding regions (Fig. 2). Functional studies will be necessary to determine if this high degree of conservation is the result of gene conversion or rather, of purifying selection driven by imperatives of gene expression. In any case, if indeed the 3' IR of the different PSA clades play a role in the control of gene expression, either the pattern of expression is variable between the clades or the cues governing this control are subtler than the primary sequence per se.

\section{Discussion and Conclusion}

Thanks to the whole genome sequencing of three Leishmania species, we could draw the complete picture of the PSA gene family. Up to now, only a few genes belonging to the Chr12 subfamily had been characterised. Also, L. braziliensis was thought not to contain any gene for that subfamily. We have proposed a nomenclature for all PSA genes (see Fig. 2 and 3). We have kept the PSA letters, even tough it is now known that these genes are expressed both in promastigote and amastigote stages and code not only for membrane proteins at the surface of the parasite but also for excreted soluble proteins. Follow the chromosome number and other symbols that refer to the phylogenetic PSA gene clusters.

PSAs are basically LRR repeats containing proteins, which are either membrane-bound at the surface of Leishmania or soluble as excreted proteins. These features suggest that this protein family could be involved in host-parasite interactions. The diversity found in PSAs, which concerns mainly the LRR domain, would be consistent with this role. This diversity is not accounted only by the sequence divergence of the LRR repeats but also by the number of these repeats present in each protein, which varies from 2 to 25 and could influence both the shape and the space occupied by PSAs, and thereby their binding properties. Two other factors of diversity in PSA reside outside the LRR domain. One concerns the Thr/Ser-rich domain, whose length varies considerably and can be totally absent in some PSAs. Although there is some indication that PSAs could be glycosylated [24,25], both the sites of glycosylation and the nature of the amino acid-glycan linkage are unknown. Most of these Thr/Ser-rich domains contain a signature present in $T$. cruzi mucins which is known to be the site of O-glycosylation. Finally, membrane attachment determinants (which result mostly in anchoring through GPI) define two destinations for PSA proteins: excretion or attachment to the parasite's surface. Although the majority are predicted to be membrane bound, PSAs predicted to be secreted are found both as strongly conserved proteins between species (PSA21 in all three species; LiPSA12A and LmPSA12A) and as species specific proteins (LiPSA12I and most LmPSA12C). This suggests that the presence of these C-terminal truncated forms of PSAs is neither fortuitous nor the products of pseudogenes, but that secretion plays a fundamental role in Leishmania.

PSA genes distribute into eight subfamilies. In view of the found orthologies, the most parsimonious scenario would be that the last common ancestor (LCA) of $L$. major, L. infantum and L. braziliensis had nine genes: one in each of chromosomes 9, 12 and 21, two on chromosome 5 and three on chromosome 31. Among the PSA subfamilies, the Chr12 subfamily is the most diversified one, at least in $L$. major and L. infantum. This is brought about by nucleotide substitutions as well as unequal gene recombination, which modulates both the number of LRRs in PSA proteins and the number of genes in the family, and generates chimeric genes. The evolution of the Chr12 subfamily of PSA genes is probably best explained by a birth-and-loss process, by which the gene family is either expanded or contracted, while PSA genes are indi- 
vidually submitted to diversifying selection. We have detected clear evidence of positive selection for the Chr12 genes in both $L$. major and $L$. infantum, while the existence of orthologs, divergent paralogs, as well as probable pseudogenes (LmjF12.1005, LinJ12_v4.0662) all point to a birth-and-loss model. We note, however, that the extremities of the coding regions and the 3' IR of the Chr12 PSA genes are all species specific (no orthologies can be inferred) and remarkably similar (some stretches are even devoid of synonymous substitutions), suggesting that some gene homogenisation might have taken place by gene conversion. In contrast to the Chr12 subfamily, the seven other subfamilies seem to evolve in a more conservative way. Not only all genes of Chr5, 9, 21 and 31 from the three Leishmania species cluster as more similar orthologs but evidence of recombination is scarce. One finds only one pair of paralogs (the L. major Chr31A subfamily), and the number of LRR repeats is the same for each group of orthologs (except one difference in Chr21 subfamily). Furthermore, diversifying selection apparently does not act on these genes, as evidenced by $d_{\mathrm{N}} / d_{\mathrm{S}}$ values well under those obtained for the Chr12 genes. We would therefore postulate the latter subfamilies to be involved in basic slowly evolving host-parasite interactions, whereas the Chr12 subfamily could be linked to more dynamic adaptative responses like optimisation of cellular infection (internalisation and maintenance in the macrophage) and escape from the immune system. The latter could also be related to the Leishmania species identities like the nature of the sand fly species infected and the clinical symptoms. Still, diversification of Chr12 genes apparently does not offer any advantage for $L$. braziliensis. At this stage, it is hard to find out what kind of adaptative role these genes could play in L. major and L. infantum, which would be dispensable for $L$. braziliensis.

Diversifying selection has been shown to drive evolution of genes involved in host-pathogen interactions. These include virulence genes, as found in Streptococci [26] and Listeria monocytogenes [27], as well as resistance genes, like the numerous plant NBS-LRR genes [28], the primate $\operatorname{Trim} 5 \alpha$ [29] genes involved in restriction of retroviruses replication and many gene classes involved in the Drosophila innate immune system [30]. Interestingly, the Listeria virulence gene inlA and the NBS-LRR genes possess LRR domains, which were shown to evolve under positive selection. In the present work, we found that in the Chr12 PSA gene subfamily of two Leishmania species, LRR domains evolve under strong positive selection. Most notably, the several residues submitted to diversifying selection are predicted to reside at the protein's surface. The importance of this evolving LRR protein interface in the parasite's life cycle awaits the determination of the precise PSA biological function and the identification of eventual host PSA interactors.
In conclusion, we have identified eight PSA subfamilies. The phylogenetic distance separating them, which is paralleled by the divergence of their LRR domains, appears large enough to reflect functional specificities. The strong conservation of the orthologs of the subfamilies 5A, 5B, 9, $21,31 \mathrm{~A}, \mathrm{~B}$ and $\mathrm{C}$ suggest that they play essential roles. Generation of knock outs and gene expression analysis of all individual PSA genes will be needed to explore the importance of these genes in the different stages of the life cycle of Leishmania, and eventually in adaptation of the parasites to their specific insect or mammalian host. In comparison to the seven subfamilies above, the PSA12 subfamily is evolving much more rapidly, (at least in $L$. major and L. infantum) as revealed by the high number of paralogs and the presence of strong diversifying selection. Sequencing of these genes in several strains of L. major and L. infantum is expected to show a high incidence of polymorphism.

\section{Methods \\ Alignment of PSA amino acid and nucleotide sequences and identification of membrane anchorage determinants}

PSA protein and DNA sequences were extracted from the L. major, L. infantum and L. braziliensis database http:// www.genedb.org/after BLAST analysis. The latest versions of the genome sequences were used, i.e. 5.2 for $L$. major, 2.0 for L. braziliensis and 3.0 (and 4.0 for chromosome 12) for $L$. infantum. Protein sequences were aligned with ClustalX 2.0 [31] followed by manual correction. These alignments were transposed to the corresponding genomic DNA alignments by protal2dna http://mobyle.pasteur.fr/ cgi-bin/MobylePortal/portal.py?form=tranalign. The presence of signal peptide and transmembrane domains was reported as found in annotations of the Leishmania genomes http://www.genedb.org/. The prediction of GPIanchorage was performed by GPI-SOM http:// gpi.unibe.ch/[32]. Genomic sequences flanking the PSA ORFs in 3' were aligned with Dialign 2.2.1 [33].

\section{Phylogenetic analysis}

Phylogenetic trees of Fig. 2 and 3 were obtained from nucleotide sequence alignments by maximum likelihood (PhyML v2.4.4) [34]. The selection of the nucleotide substitution model (and eventually of amino acids substitution) was determined by ModelGenerator http:// distributed.cs.nuim.ie/multiphyl.php[35]. The HKY+I+G (Fig. 2) and TN93+G+I (Fig. 3) nucleotide models were used, with the base frequency, Ts/Tv ratio, and gamma distribution parameter estimated by PhyML. These trees were compared to those obtained by PhyML from the corresponding amino acid sequences (WAG $+G$ model of amino acids substitution) or with a distance method implemented in MEGA v3.1 [36] using both DNA (TN93) or amino acids (JTT model) alignments. Occasionally (see text in Results section), a codon model $\left(d_{\mathrm{S}^{\prime}}\right.$ number of 
synonymous substitutions per synonymous sites; NeiGojobori method, JC model) as implemented in MEGA was used to calculate distance matrix. Trees were annotated and visualised by Treedyn (v194.3) [37].

\section{Positive selection}

One level of positive selection analysis involved calculation of $d_{\mathrm{N}} / d_{\mathrm{S}}$ ratios between pairs of sequences (MEGA, Nei-Gojobori method, JC model). $\omega=d_{\mathrm{N}} / d_{\mathrm{S}}<1$ corresponds to purifying (negative) selection, $\omega=1$ to neutral evolution (absence of selection), and $\omega>1$ indicating adaptive evolution (positive selection). The maximum likelihood based method implemented in the codeml program of the PAML package (v3.15) [38] was used to detect positive selection as well as identifying positively selected sites, when the number of related sequences was sufficiently high. To detect positive selection, we performed likelihood ratio test (LRT) analysis between three pairs of models to determine whether particular models provided a significantly better fit to the data: M1a ("Nearly-Neutral" model) vs. M2a ("PositiveSelection" model), M7 ("beta" model) vs. M8 ("beta\& $\omega$ " model) and M8a vs. M8. Only models M2a and M8 allow for site categories evolving under positive selection (see Yang et al. 2005 [39] for further details on the models). Positive selection is inferred when the $(2 \Delta \lambda)$ statistic is greater than critical values 1 ) of the Chi square distribution for a degree of freedom of 2 (M1a/M2a. and M7/M8 comparisons) or 2) of a 50:50 mixture of point mass 0 and Chi square (critical values are 2.71 at $5 \%$ and 5.41 at $1 \%$; M8a/M8 comparison). Only predicted positively selected sites with posterior BEB (Bayes empirical Bayes) probabilities greater than 0.95 or 0.90 were retained.

\section{Authors' contributions}

$\mathrm{AD}$ carried out the data mining, the sequence alignments, bioinformatic analysis and wrote the manuscript. ALB conceived the study and participated in writing the manuscript. All authors read and approved the final manuscript.

\section{Additional material}

\section{Additional file 1}

DNA sequence alignment of all PSA genes.T/S- rich, TM/GPI anchor and LRR domains (except the most C-terminal LRR repeat) were excluded from the alignment, as well as a unique repeat of the LRR-like domain in LinJ12_v4.0670.

Click here for file

[http://www.biomedcentral.com/content/supplementary/14712148-8-292-S1.txt]

\section{Additional file 2}

Amino acid sequence alignment of the LRR domain of proteins of cluster 5A.

Click here for file

[http://www.biomedcentral.com/content/supplementary/1471-

2148-8-292-S2.txt]

\section{Additional file 3}

Amino acid sequence alignment of the LRR domain of proteins of cluster $5 B$.

Click here for file

[http://www.biomedcentral.com/content/supplementary/1471-

2148-8-292-S3.txt]

\section{Additional file 4}

Amino acid sequence alignment of the LRR domain of proteins of cluster 9.

Click here for file

[http://www.biomedcentral.com/content/supplementary/1471-

2148-8-292-S4.txt]

\section{Additional file 5}

Amino acid sequence alignment of the LRR domain of proteins of cluster 21.

Click here for file

[http://www.biomedcentral.com/content/supplementary/1471-

2148-8-292-S5.txt]

\section{Additional file 6}

Amino acid sequence alignment of the LRR domain of proteins of cluster 31A.

Click here for file

[http://www.biomedcentral.com/content/supplementary/1471-

2148-8-292-S6.txt]

\section{Additional file 7}

Amino acid sequence alignment of the LRR domain of proteins of cluster $31 B$.

Click here for file

[http://www.biomedcentral.com/content/supplementary/1471-

2148-8-292-S7.txt]

\section{Additional file 8}

Amino acid sequence alignment of the LRR domain of proteins of cluster 31C.

Click here for file

[http://www.biomedcentral.com/content/supplementary/1471-

2148-8-292-S8.txt]

\section{Additional file 9}

Amino acid sequence alignment of the LRR domain of all PSA proteins of Chr12. Some repeats unique to certain proteins were deleted.

Click here for file

[http://www.biomedcentral.com/content/supplementary/14712148-8-292-S9.txt] 


\section{Additional file 10}

Structure of the PSA gene cluster on chromosome 12 of $\mathrm{L}$. major. The indicated LmjF12.xxxx PSA genes (rectangular boxes) and the intervening type A (LmjF12.0880, 0885, 0905, 0995, 1015) and B

(LmjF12.0715, 0860, 0800, 0820, 0840, 0880, 0895, 0930, 0950, $0970,1030,1050)$ paralogs form a single uninterrupted cluster. PSA genes belonging to the same phylogenetic sub-clusters are represented by the same colour. Arrows link highly similar genes that probably arose by duplication of a cluster of five genes.

Click here for file

[http://www.biomedcentral.com/content/supplementary/14712148-8-292-S10.pdf]

\section{Additional file 11}

Alignment of 3 ' intergenic region of PSA genes from clades $5 A, B, 9$, $21,31 A, B, C$. Sequences are listed according to their clade in the above order. Alignments were performed only between orthologs. 3' IR region of gene LbrM31_V2.1670 is not determined in the genome database.

Click here for file

[http://www.biomedcentral.com/content/supplementary/1471-

2148-8-292-S11.txt]

\section{Additional file 12}

Alignment of 3' intergenic region of PSA genes from clade 12 (L. infantum and L. major).

Click here for file

[http://www.biomedcentral.com/content/supplementary/14712148-8-292-S12.txt]

\section{Additional file 13}

DNA sequence alignment of the LRR domain of all PSA genes of Chr12. DNA alignment transformed from that of Additional file 9 and used to infer phylogenetic tree of Fig. 3.

Click here for file

[http://www.biomedcentral.com/content/supplementary/14712148-8-292-S13.txt]

\section{Additional file 14}

DNA sequence alignment of a subset of Chr12 PSA genes used for recombination analysis of gene LmjF12.0990 in Fig.5.

Click here for file

[http://www.biomedcentral.com/content/supplementary/14712148-8-292-S14.txt]

\section{Additional file 15}

DNA sequence alignment of a subset of Chr12 PSA genes used for recombination analysis of gene LmjF12.0960 in Fig.5.

Click here for file

[http://www.biomedcentral.com/content/supplementary/14712148-8-292-S15.txt]

\section{Additional file 16}

DNA sequence alignment of Chr12 PSA genes used for positive selection analysis in Fig.7.

Click here for file

[http://www.biomedcentral.com/content/supplementary/14712148-8-292-S16.txt]

\section{Acknowledgements}

We thank F.Chevenet for advice in running the Treedyn software, A. Kajava for structure modelling of PSA LRRs and E. Desmarais for critical reading of the manuscript.

\section{References}

I. Schlein Y: Leishmania and Sandflies: interactions in the life cycle and transmission. Parasitol Today 1993, 9(7):255-258.

2. Banuls AL, Hide M, Prugnolle F: Leishmania and the leishmaniases: a parasite genetic update and advances in taxonomy, epidemiology and pathogenicity in humans. Adv Parasitol 2007, 64:1-109.

3. Frommel TO, Button LL, Fujikura Y, McMaster WR: The major surface glycoprotein (GP63) is present in both life stages of Leishmania. Mol Biochem Parasitol 1990, 38(I):25-32.

4. Jimenez-Ruiz A, Boceta C, Bonay P, Requena JM, Alonso C: Cloning, sequencing, and expression of the PSA genes from Leishmania infantum. Eur J Biochem I998, 25 I (I-2):389-397.

5. Ilg T: Proteophosphoglycans of Leishmania. Parasitol Today 2000 , I6(I I):489-497.

6. Rittig MG, Bogdan C: Leishmania-host-cell interaction: complexities and alternative views. Parasitol Today 2000, 16(7):292-297.

7. Santarem N, Silvestre R, Tavares J, Silva M, Cabral S, Maciel J, Cordeiro-da-Silva $A$ : Immune response regulation by leishmania secreted and nonsecreted antigens. J Biomed Biotechnol 2007, 2007(6):85I54.

8. Olivier M, Gregory DJ, Forget G: Subversion mechanisms by which Leishmania parasites can escape the host immune response: a signaling point of view. Clin Microbiol Rev 2005, I 8(2):293-305.

9. Handman E, Osborn AH, Symons F, van Driel R, Cappai R: The Leishmania promastigote surface antigen 2 complex is differentially expressed during the parasite life cycle. Mol Biochem Parasitol 1995, 74(2): 189-200.

10. Beetham JK, Donelson JE, Dahlin RR: Surface glycoprotein PSA (GP46) expression during short- and long-term culture of Leishmania chagasi. Mol Biochem Parasitol 2003, I 3 I (2): I09-I 17.

II. Lincoln LM, Ozaki M, Donelson JE, Beetham JK: Genetic complementation of Leishmania deficient in PSA (GP46) restores their resistance to lysis by complement. Mol Biochem Parasitol 2004, 137(I): 185-189.

12. Pizarro-Cerda J, Cossart P: Bacterial adhesion and entry into host cells. Cell 2006, I 24(4):7|5-727.

13. Seepersaud R, Hanniffy SB, Mayne P, Sizer P, Le Page R, Wells JM: Characterization of a novel leucine-rich repeat protein antigen from group B streptococci that elicits protective immunity. Infect Immun 2005, 73(3): I67I-I683.

14. Athman R, Philpott D: Innate immunity via Toll-like receptors and Nod proteins. Curr Opin Microbiol 2004, 7(I):25-32.

15. McHale L, Tan X, Koehl P, Michelmore RW: Plant NBS-LRR proteins: adaptable guards. Genome Biol 2006, 7(4):2I2.

16. Lohman KL, Langer PJ, McMahon-Pratt D: Molecular cloning and characterization of the immunologically protective surface glycoprotein GP46/M-2 of Leishmania amazonensis. Proc Nat Acad Sci USA 1990, 87(21):8393-8397.

17. Myung KS, Beetham JK, Wilson ME, Donelson JE: Comparison of the post-transcriptional regulation of the mRNAs for the surface proteins PSA (GP46) and MSP (GP63) of Leishmania chagasi. J Biol Chem 2002, 277(19):16489-16497.

18. Peacock CS, Seeger K, Harris D, Murphy L, Ruiz JC, Quail MA, Peters $\mathrm{N}$, Adlem E, Tivey A, Aslett M, et al:: Comparative genomic analysis of three Leishmania species that cause diverse human disease. Nat Genet 2007, 39(7):839-847.

19. Ivens AC, Peacock CS, Worthey EA, Murphy L, Aggarwal G, Berriman M, Sisk E, Rajandream MA, Adlem E, Aert R, et al.: The genome of the kinetoplastid parasite, Leishmania major. Science 2005, 309(5733):436-442.

20. Kajava AV: Structural diversity of leucine-rich repeat proteins. J Mol Biol 1998, 277(3):5 I9-527.

21. Lole KS, Bollinger RC, Paranjape RS, Gadkari D, Kulkarni SS, Novak NG, Ingersoll R, Sheppard HW, Ray SC: Full-length human immunodeficiency virus type I genomes from subtype C-infected seroconverters in India, with evidence of intersubtype recombination. J Virol 1999, 73(I): 152-160. 
22. Anisimova M, Nielsen R, Yang Z: Effect of recombination on the accuracy of the likelihood method for detecting positive selection at amino acid sites. Genetics 2003, I64(3): I229-1236.

23. Kajava AV, Kobe B: Assessment of the ability to model proteins with leucine-rich repeats in light of the latest structural information. Protein Sci 2002, I I(5): 1082-1090.

24. Kahl LP, McMahon-Pratt D: Structural and antigenic characterization of a species- and promastigote-specific Leishmanic mexicana amazonensis membrane protein. J Immunol 1987 I38(5): | 587-| 595.

25. Murray PJ, Spithill TW, Handman E: The PSA-2 glycoprotein complex of Leishmania major is a glycosylphosphatidylinositol-linked promastigote surface antigen. J Immunol 1989, I 43( I 2):422I-4226.

26. Anisimova M, Bielawski J, Dunn K, Yang Z: Phylogenomic analysis of natural selection pressure in Streptococcus genomes. BMC Evol Biol 2007, 7:154.

27. Orsi RH, Ripoll DR, Yeung M, Nightingale KK, Wiedmann $M$ Recombination and positive selection contribute to evolution of Listeria monocytogenes inlA. Microbiology 2007, I 53(Pt 8):2666-2678.

28. Lehmann P: Structure and evolution of plant disease resistance genes. J Appl Genet 2002, 43(4):403-4I4.

29. Sawyer SL, Wu LI, Emerman M, Malik HS: Positive selection of primate TRIM5alpha identifies a critical species-specific retroviral restriction domain. Proc Natl Acad Sci USA 2005, I 02(8):2832-2837.

30. Sackton TB, Lazzaro BP, Schlenke TA, Evans JD, Hultmark D, Clark AG: Dynamic evolution of the innate immune system in Drosophila. Nat Genet 2007, 39( I 2): I 46 I-I 468.

31. Thompson JD, Gibson T], Plewniak F, Jeanmougin F, Higgins DG: The CLUSTAL_X windows interface: flexible strategies for multiple sequence alignment aided by quality analysis tools. Nucleic Acids Res 1997, 25(24):4876-4882.

32. Fankhauser N, Maser P: Identification of GPI anchor attachment signals by a Kohonen self-organizing map. Bioinformatics 2005, 2 I(9): 1846- 852.

33. Morgenstern B: DIALIGN 2: improvement of the segment-tosegment approach to multiple sequence alignment. Bioinformatics 1999, I5(3):21 I-218.

34. Guindon S, Gascuel O: A simple, fast, and accurate algorithm to estimate large phylogenies by maximum likelihood. Syst Biol 2003, 52(5):696-704.

35. Keane TM, Creevey CJ, Pentony MM, Naughton TJ, McLnerney JO: Assessment of methods for amino acid matrix selection and their use on empirical data shows that ad hoc assumptions for choice of matrix are not justified. BMC Evol Biol 2006, 6:29.

36. Kumar S, Tamura K, Nei M: MEGA3: Integrated software for Molecular Evolutionary Genetics Analysis and sequence alignment. Brief Bioinform 2004, 5(2): 150-163.

37. Chevenet F, Brun C, Banuls AL, Jacq B, Christen R: TreeDyn: towards dynamic graphics and annotations for analyses of trees. BMC Bioinformatics 2006, 7:439.

38. Yang Z: PAML: a program package for phylogenetic analysis by maximum likelihood. Comput Appl Biosci 1997, I3(5):555-556.

39. Yang Z, Wong WS, Nielsen R: Bayes empirical bayes inference of amino acid sites under positive selection. Mol Biol Evol 2005, 22(4): $1107-1|1|$
Publish with Bio Med Central and every scientist can read your work free of charge

"BioMed Central will be the most significant development for disseminating the results of biomedical research in our lifetime. "

Sir Paul Nurse, Cancer Research UK

Your research papers will be:

- available free of charge to the entire biomedical community

- peer reviewed and published immediately upon acceptance

- cited in PubMed and archived on PubMed Central

- yours - you keep the copyright
BioMedcentral 